\title{
Obesity-programmed mice are rescued by early genetic intervention
}

\author{
Viviana F. Bumaschny, ${ }^{1,2}$ Miho Yamashita, ${ }^{3}$ Rodrigo Casas-Cordero, ${ }^{1}$ Verónica Otero-Corchón, ${ }^{3}$ \\ Flávio S.J. de Souza, ${ }^{1,4}$ Marcelo Rubinstein,1,4 and Malcolm J. Low ${ }^{3}$

\begin{abstract}
1Instituto de Investigaciones en Ingeniería Genética y Biología Molecular, Consejo Nacional de Investigaciones Científicas y Técnicas, Buenos Aires, Argentina. ${ }^{2}$ Departamento de Fisiología y Biofísica, Facultad de Medicina, Universidad de Buenos Aires, Buenos Aires, Argentina ${ }^{3}$ Department of Molecular and Integrative Physiology, University of Michigan, Ann Arbor, Michigan, USA. ${ }^{4}$ Departamento de Fisiología, Biología Molecular y Celular, Facultad de Ciencias Exactas y Naturales, Universidad de Buenos Aires, Buenos Aires, Argentina.
\end{abstract}

\begin{abstract}
Obesity is a chronic metabolic disorder affecting half a billion people worldwide. Major difficulties in managing obesity are the cessation of continued weight loss in patients after an initial period of responsiveness and rebound to pretreatment weight. It is conceivable that chronic weight gain unrelated to physiological needs induces an allostatic regulatory state that defends a supranormal adipose mass despite its maladaptive consequences. To challenge this hypothesis, we generated a reversible genetic mouse model of early-onset hyperphagia and severe obesity by selectively blocking the expression of the proopiomelanocortin gene (Pomc) in hypothalamic neurons. Eutopic reactivation of central POMC transmission at different stages of overweight progression normalized or greatly reduced food intake in these obesity-programmed mice. Hypothalamic Pomc rescue also attenuated comorbidities such as hyperglycemia, hyperinsulinemia, and hepatic steatosis and normalized locomotor activity. However, effectiveness of treatment to normalize body weight and adiposity declined progressively as the level of obesity at the time of Pomc induction increased. Thus, our study using a novel reversible monogenic obesity model reveals the critical importance of early intervention for the prevention of subsequent allostatic overload that auto-perpetuates obesity.
\end{abstract}

\section{Introduction}

Obesity is a chronic metabolic disorder that affects more than 500 million adults and 43 million children under the age of 5 worldwide (1). Overweight predisposes individuals to numerous comorbidities, including type 2 diabetes mellitus, hypertension, cardiovascular disease, and certain forms of cancer (2). The causes of obesity are complex, because environmental, social, and genetic factors converge to produce a condition in which energy intake consistently exceeds energy expenditure, leading to the storage of excess calories as fat.

Unlike homeostatic processes that operate around narrowly defined set points defended solely by the autonomic nervous and endocrine systems, body weight regulation and fat storage are allostatic metabolic processes by which voluntary actions, including foraging and food intake, are engaged in anticipation of future caloric deficits including foraging and food intake. Because body growth is an enduring process across the first stages of a mammal's life, the allostatic state is evident from early development of the fetus and continues postnatally until completion of sexual maturation. Thereafter, body weight is typically defended around a relatively more stable set point (3), with the exception of intervening physiological conditions such as pregnancy, lactation, or hibernation. These latter states reengage the allostatic energy balance system to trigger short-term feed-forward mechanisms that promote an increase in food intake, fat accumulation, and body weight. All of these parameters revert to previous values once less-demanding environmental conditions allow reduced caloric intake. Similarly, voluntary overfeeding can lead to a great increase in body weight that gradually returns to preexisting levels when normal feeding is

Authorship note: Marcelo Rubinstein and Malcolm J. Low are co-senior authors. Conflict of interest: The authors have declared that no conflict of interest exists. Citation for this article: J Clin Invest. 2012;122(11):4203-4212. doi:10.1172/JCI62543. resumed $(4,5)$. However, this is not generally true in obese patients, who demonstrate an inability to completely achieve a normal weight and exhibit high recidivism after dieting, in large part due to compensatory and persistent reductions in metabolic rate (6-8). These problems worsen in extreme obesity $\left(\mathrm{BMI}>40 \mathrm{~kg} / \mathrm{m}^{2}\right)$, a condition that is increasing alarmingly $(9,10)$. Diet-induced obesity in rats and mice can also permanently elevate body weight set point after the animals are switched back to normal chow (11-13). However, unlike the allostatic changes in metabolism that occur during pregnancy or hibernation to promote "viability through change" (14), chronic overfeeding unrelated to physiological needs generates an allostatic state that slowly leads to obesity and is ultimately maladaptive to the health of an organism.

The brain is a main regulator of energy balance and receives peripheral signals of energy status in the form of circulating nutrients and hormones, such as leptin, combined with afferent neural signals relayed through the vagus nerve (15). Two populations of neurons within the arcuate nucleus (Arc) of the hypothalamus sense and respond to most of these signals (16). Orexigenic agouti-related peptide (AgRP) and neuropeptide Y (NPY) neurons are inhibited by leptin, while proopiomelanocortin (POMC) and cocaine- and amphetamine-related transcript (CART) neurons are stimulated by leptin. Pomc is highly expressed in the pituitary gland and hypothalamus of all vertebrates (17) by means of two independent cis-acting enhancer modules; a proximal promoter that controls pituitary Pomc expression and a distal module that is necessary for hypothalamic Pomc expression (18). There is also a small number of neurons in the nucleus tractus solitarius (NTS) of the brainstem that express much lower levels of Pomc mRNA and whose physiological function is poorly understood. Among hypothalamic POMC products are the anorectic melanocortin peptides $\alpha$ - and $\beta$-MSH and the opioid peptide $\beta$-endorphin, which are released in response to metabolic peripheral signals $(19,20)$. 
Genetic inactivation of POMC in humans and rodents results in severe hyperphagia, early-onset obesity, and profound adrenal insufficiency $(21,22)$, whereas mutations in the gene encoding central melanocortin receptor $4(\mathrm{MC} 4 \mathrm{R})$ are the most common monogenic disorders causing obesity (16).

Because of the high prevalence of overweight and the severity of its comorbidities $(1,2)$, it is imperative to understand the factors associated with treatment refractoriness. Our principal hypothesis is that energy homeostasis can be disturbed acutely within a certain range without major consequences for the organism. However, when chronic perturbations occur, such as severe hyperphagia or sustained low energy expenditure, the elastic limits of the regulatory system are exceeded and normal weight can no longer be achieved due to persistent adaptations. To test this hypothesis, we generated a reversible knockout mouse model of early-onset extreme obesity by inhibiting Pomc expression in the hypothalamus through a genetic switch that allows subsequent reactivation of gene expression. These mice develop obesity while consuming a standard low-fat chow, and therefore the genetic model avoids the conundrum of permanent changes in dopaminergic reward centers induced by high-fat or high-sucrose diets, even after return to food with low hedonic value (23), as typically occurs in dietinduced obesity models. We rescued hypothalamic Pomc in mice with different degrees of overweight and found marked improvements in food intake, body weight, fat deposits, and fasting glucose even in cases of extreme obesity. However, increasing levels of overweight at the time of genetic rescue progressively reduced the ability of mice to achieve a normal body weight.

\section{Results}

Generation of a reversible central Pomc-knockout mouse model of obesity.

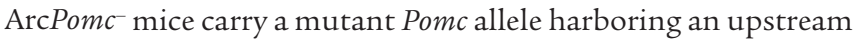
loxP-flanked neo cassette that selectively prevents neuronal transcription in the hypothalamic Arc but not in pituitary cells (Figure 1, A and B). The lack of hypothalamic Pomc expression in arcPomc ${ }^{-1-}$ mice led to early-onset extreme obesity, readily detected by age 5 weeks $\left(P<0.001\right.$ versus $\mathrm{Pomc}^{+/+}$and $\mathrm{arcPomc}^{+/-}$littermates, 1-way ANOVA [OWA], Figure 1C). However, compound arcPomc ${ }^{-1-}$ mice carrying a Pomc-EGFP transgene (19) expressed the EGFP reporter molecule in the same number, and with a similar morphology and spatial location, of neurons within the Arc as $\mathrm{Pomc}^{+/+}$mice (Figure 1B). The EGFP-positive neurons also projected widely throughout the brain to areas normally innervated by POMC neurons (data not shown). These results indicate that a subpopulation of Arc neurons is differentiated with the transcriptional machinery capable of normally activating Pomc and projects to relevant target areas despite the absence of POMC-derived peptides throughout prenatal development and into postnatal life. ArcPomc ${ }^{-1-}$ mice were hyperphagic (Figure 1D) and had no detectable hypothalamic Pomc mRNA (Figure 1E), but NTS Pomc mRNA levels were reduced only to $44 \% \pm 3 \%$ $(n=6)$ of wild-type levels. In contrast, body weight and daily food intake were normal in arcPomc ${ }^{+/-}$mice despite the fact that hypothalamic Pomc mRNA levels were 50\% of those in their $\mathrm{Pomc}^{+/+}$ siblings (Figure 1, C-E).

Excision of the neo cassette by a cre recombinase transgene at preimplantation embryonic stages (24) reestablished central Pomc expression, demonstrating that the phenotype is fully reversible (Figure 2A). Rescued arcPomcrue/rcue mice showed normal hypothalamic Pomc mRNA levels: $90 \% \pm 11 \%$ in males and $84 \% \pm 13 \%$ in females $\left(P=0.29\right.$ and $P=0.21$ respectively, $t$ tests vs. $P o m c^{+/+}$mice). Consequently, germline-rescued mice exhibited normal body weight and normal daily food intake (Figure 2, B and C).

Pomc rescue at different stages. To investigate whether postnatal recovery of neuronal Pomc expression could normalize body weight in mice that developed and grew through weaning in the absence of central POMC peptides, we obtained compound $\mathrm{arcPomc}^{-1-}$;Cre-ERT mice after successive crossing of $\operatorname{arcPomc}^{+/-}$mice with transgenic mice expressing a tamoxifen-inducible (TAM-inducible) cre recombinase (Cre-ERT; ref. 25). The Cre-ERT transgene driven by a ubiquitously active CAAG promoter was chosen for these studies because it produced TAM-dependent recombination of a loxP-STOP reporter allele in the majority of cells in virtually all tissues examined (25). The cellular specificity of Pomc reactivation by Cre-ERT is due to the endogenous Pomc regulatory elements in the knockin arcPomc allele. At P25, in juvenile arcPomc ${ }^{-1}$ :Cre-ERT mice that were still lean, TAM-induced rescue of Pomc expression completely prevented obesity in both sexes (Figure 3A). Conversely, arcPomc ${ }^{-1}$ mice treated with TAM and arcPomc ${ }^{-1-}$ :Cre-ERT mice treated with vehicle grew significantly heavier than $\mathrm{Pomc}^{+/+}$or $\mathrm{Pomc}^{+/+}$:Cre-ERT control mice $(P<0.001)$. TAM administration at P60 to obese

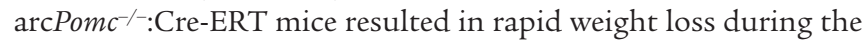
first 3 weeks and, following a plateau, resumption of weight gain at the same rate as that of $\mathrm{Pomc}^{+/+}$littermates (Figure 3B; from week 12 to 24; repeated measures ANOVA [RMA], genotype $\times$ time effect, $P>0.05)$. Despite their improvement, male mice rescued at P60 remained heavier than $\mathrm{Pomc}^{+/+}$controls $(P<0.01)$. To further study the ability of the energy balance homeostat to restore body weight in cases of extreme obesity, we rescued Pomc expression in P180 arcPomc ${ }^{-1-}$ :Cre-ERT mice (Figure 3, C and D). Notably, the overweight of female mice treated at P180 decreased dramatically from approximately $164 \%$ to $41 \%$ of $\mathrm{Pomc}^{+/+}$controls compared with a more modest reduction from approximately $109 \%$ to $59 \%$ of controls in males (Figure 3C). Although P180-rescued mice showed significant weight loss, they remained more overweight than mice rescued at $\mathrm{P} 60$. Moreover, arcPomc ${ }^{-1-}$ :Cre-ERT male mice rescued at P180, after reaching a nadir, regained weight faster than $\mathrm{Pomc}^{+/+}$ controls (RMA, genotype $\times$ time effect, $P<0.01$ ). Together, these results show that Pomc expression can be restored eutopically in Arc neurons of weanling mice, leading to complete normalization of subsequent growth and body weight. However, the improvement in body weight is progressively attenuated, as the age of Pomc rescue is delayed from weaning to adulthood.

The age and sex differences in treatment results cannot be simply attributed to variation in recombination efficiency of the mutant Pomc alleles, since the percentage and rostro-caudal distribution of immunoreactive arcuate POMC neurons was similar among all groups ( 75\%) after TAM (Supplemental Figure 1 and Supplemental Table 1; supplemental material available online with this article; doi:10.1172/JCI62543DS1). However, there was a trend toward decreased hypothalamic Pomc mRNA levels as the age of TAM treatment was delayed (Supplemental Table 1). An inverse correlation between hypothalamic Pomc mRNA and obesity scores could be the result of increased leptin resistance to signaling mechanisms that normally promote Pomc expression (26).

To further test our hypothesis that the preceding development of obesity was directly responsible for the attenuated phenotypic response to Pomc restoration at P60 compared with P25 in male mice, we studied additional cohorts of $\mathrm{arcPomc}^{--}$and $\operatorname{arcPomc}^{-1}:$ Cre-ERT mice whose body weight was matched to that 
A

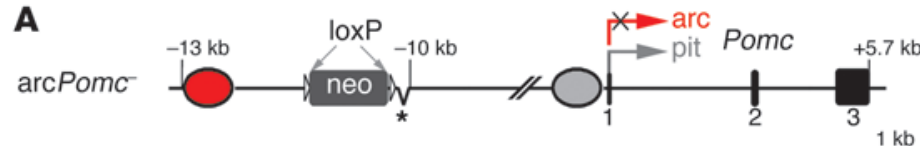

B

$\operatorname{arcPomc} c^{+/}$

$1 \mathrm{~kb}$
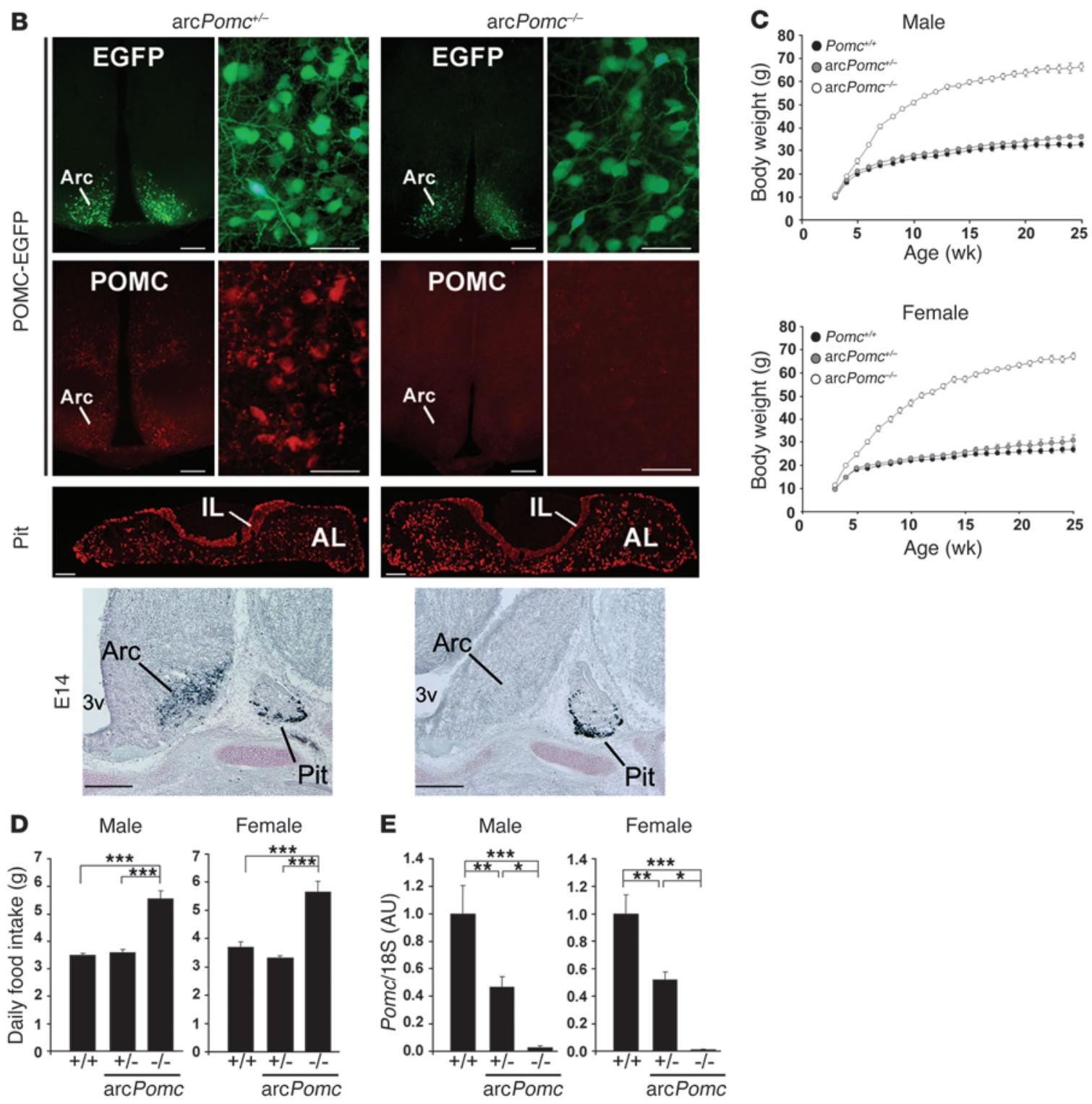

Figure 1

ArcPomc $^{-/-}$mice are obese and hyperphagic due to an impairment in hypothalamic Pomc expression. (A) ArcPomc ${ }^{-}$allele contains an insertion of a neomycin resistance cassette (neo), flanked by loxP sites, interrupting Pomc neuronal enhancer activity. Red oval, neuronal Pomc enhancer 1 (nPE1); asterisk, deletion of nPE2 (18); gray oval, Pomc promoter/pituitary enhancer; black rectangles, Pomc exons; arc and pit arrows, arcuate and pituitary Pomc transcription, respectively; X, inhibition of arcuate Pomc transcription. (B) Sections of arcPomc $\mathrm{c}^{+/-}$and arcPomc $\mathrm{c}^{-/-}$mice. Top two rows (POMC-EGFP): Mice transgenic for POMC-EGFP (19); upper panels show endogenous EGFP in POMC cells of coronal brain sections, and lower panels correspond to ACTH immunofluorescence (red, "POMC") of the same slices. Arc, arcuate neurons. Third row (Pit): Pituitaries with ACTH immunofluorescent corticotrophs of the anterior lobe (AL) and melanotrophs of the intermediate lobe (IL). Fourth row (E14): Sagittal sections of E14 mice showing ACTH immunostaining. Pit, pituitary; 3v, third ventricle. Scale bars: $200 \mu \mathrm{m}$ for lower- and 50 um for higher-magnification photomicrographs. (C) Body weight curves of $\mathrm{Pomc}^{+/+}$, arcPomc ${ }^{+/-}$, and arcPomc ${ }^{-/-}$mice. ArcPomc $\mathrm{C}^{-/-}$mice are obese (RMA, genotype $\times$time effect: $P<0.001$ for arcPomc ${ }^{-/-}$vs. Pomc ${ }^{+/+}$and vs. arcPomc ${ }^{+-}$for both sexes). (D) Average daily food intake measured during 3 consecutive weeks at 5-6 months of age. (E) Hypothalamic Pomc mRNA expression normalized to $18 \mathrm{~S}$ rRNA, relative to Pomc ${ }^{+/+}$, in arbitrary units (AU). (D and E) $n=4-6$ for each group. Error bars correspond to SEM. ${ }^{*} P<0.05,{ }^{\star \star} P<0.01,{ }^{\star \star \star} P<0.001$ (OWA). 


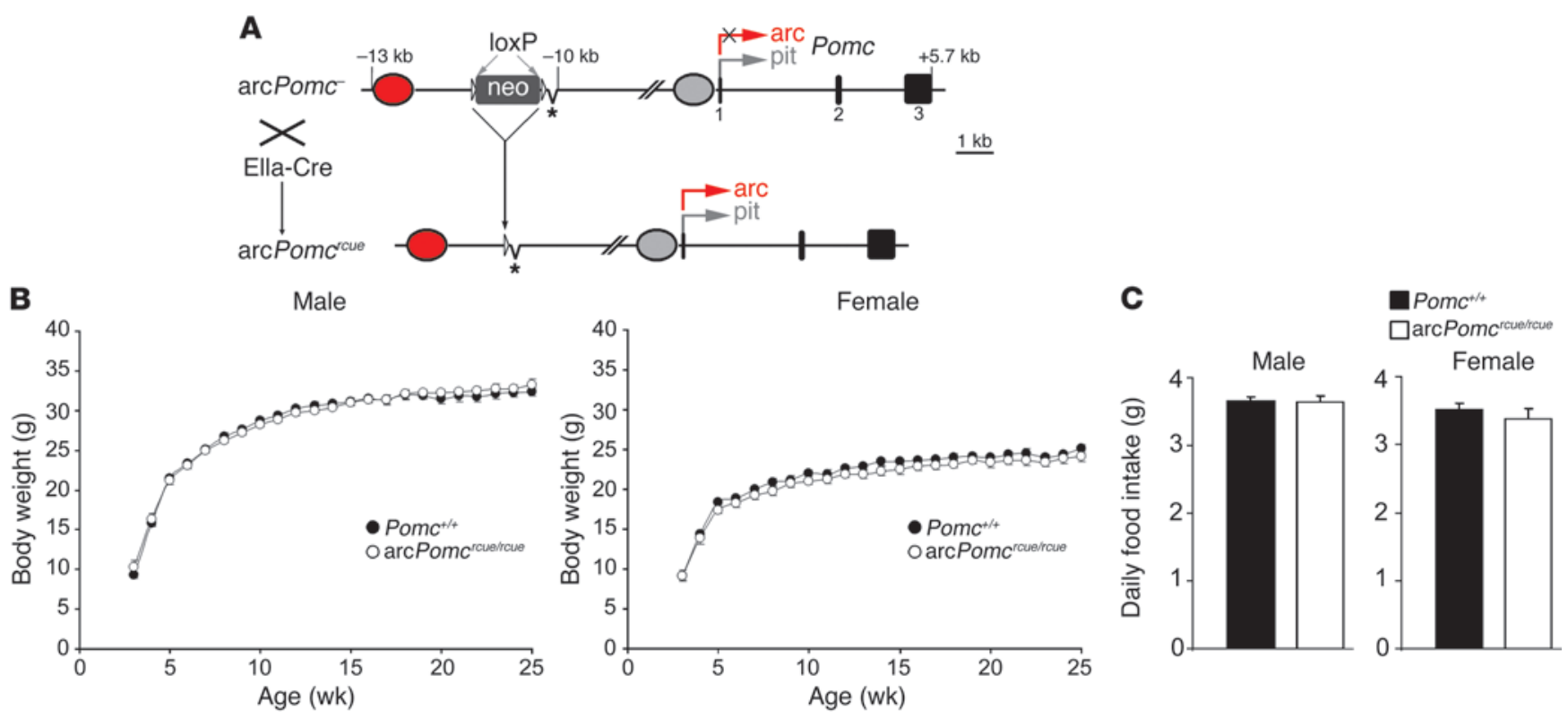

Figure 2

Arcuate Pomc rescue in the embryo normalizes food intake and body weight. (A) The arcPomc- line was crossed with an Ella-Cre line to achieve germline deletion of the floxed neomycin (neo) cassette. After successive crosses, arcPomc ${ }^{+/+}$mice (homozygous for neo deletions but negative for the Cre transgene) were obtained. Red oval, neuronal Pomc enhancer 1 (nPE1); asterisk, deletion of nPE2 (18); gray oval, Pomc pituitary promoter/ enhancer; black rectangles, Pomc exons; arc and pit arrows, arcuate and pituitary Pomc transcription, respectively; X, inhibition of arcuate Pomc transcription. (B) Body weight growth curves were completely normalized after neo removal in arcPomcrcue/rcue mice (genotype effect: $F[1,14]=0.02$, $P=0.88$ for males and $\mathrm{F}[1,9]=1.54, P=0.25$ for females with respect to arcPomcrcue/rcue littermates; RMA). Error bars correspond to SEM. (C) Daily food intake was also normalized after neo removal in $\operatorname{arcPomc^{+/+}}$ mice (genotype effect: $\mathrm{F}[1,14]=0.02, P=0.90$ for males and $\mathrm{F}[1,9]=0.52$, $P=0.49$ for females). Data correspond to averages of daily food intake measured during 3 consecutive weeks by the age of $5-6$ months.

of wild-type controls by food restriction from weaning until P60 (Figure 3E). After TAM treatment, the mice had free access to chow. The $\operatorname{arcPomc}^{-1}$ :Cre-ERT mice that had been prevented from becoming overweight by restriction of their food intake continued to have completely normal body weight growth and ad libitum food intake relative to controls for the 10 weeks after Pomc rescue. Conversely, arcPomc $\mathrm{mic}^{-/-}$mice reverted to a hyperphagic state 2 weeks after TAM treatment, with accelerated weight gain similar in trajectory to that of naive mutant mice. Hypothalamic Pomc mRNA levels in the rescued arcPomc ${ }^{-1-}$ :Cre-ERT mice were $47 \% \pm 10 \%$ $(n=3)$ of the levels in the corresponding lean wild-type mice. These results demonstrate that the body weight, but not age, at the time of arcuate Pomc rescue conditions the levels of food intake that will maintain subsequent body weight at differential set points, as observed when comparing ad libitum fed versus food-restricted P60 Pomc-rescued mice (Figure 3, B, left panel, and E).

ArcPomc ${ }^{-1-}$ :Cre-ERT females, regardless of treatment age, completely normalized their food intake, as did males treated with TAM at P25 (Figure 4A). Although previously obese P60- and P180-rescued males also greatly reduced their food intake, they still exhibited a significant residual hyperphagia (12\%-20\% greater than controls; Figure 4, A and B). Food intake fluctuations paralleled the alterations in body weight, since all Pomc-rescued mice were profoundly hypophagic during the first 2-3 weeks after TAM administration (Figure 4, B and C), causing them to lose weight during the same period (see Figure 3, B and C, and Figure 4C).

Assessment of changes in fat mass, locomotor activity, and energy expenditure after Pomc rescue. Since persistent overweight despite normalization of food intake might be attributed to decreased energy expenditure, a separate cohort of P60 mice was examined by indirect calorimetry before and after Pomc rescue. Body composition measured by NMR prior to TAM treatment demonstrated that the increased body weight of $\operatorname{arcPomc}^{-/-}$mice was primarily due to excessive fat; however lean body mass was also significantly increased $(\mathrm{OWA} \mathrm{F}[2,17]=21.3, P<0.0001$ females; $\mathrm{F}[2,15]=8.3$, $P<0.001$ males), consistent with their approximately $5 \%$ increased body length (data not shown) (Figure 5A and Supplemental Figure 2A). However, the weight loss induced by Pomc recovery in

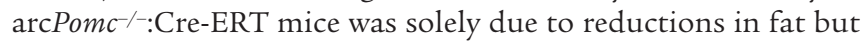
not lean mass. Obese mice of both sexes showed reduced locomotor activity before treatment that was normalized after Pomc rescue (Figure 5B and Supplemental Figure 2B). In contrast, obese mice showed an approximately $10 \%$ reduction in oxygen consumption corrected for lean body mass that was unchanged after Pomc rescue (Figure 5C and Supplemental Figure 2C). Together, these results suggest that the body weight improvement in rescued arcPomc ${ }^{-1-}$ :Cre-ERT mice is determined by the restoration of melanocortin anorectic activity that follows the recovery of Pomc expression. The residual overweight of male $\operatorname{arcPomc}^{-1-}$ :Cre-ERT mice rescued at P60 and P180 may have been caused by a small but persistent degree of hyperphagia, while female P60- and P180-rescued mice probably could not completely normalize their body weight because of persistently reduced energy expenditure.

Obesity comorbidities were reduced after Pomc restoration. Secondary conditions, such as ectopic fat deposition and diabetes, are major comorbidities in obesity. Therefore, we evaluated the improvement of several metabolic parameters in hypothalamic Pomc-rescued mice. Visceral (gonadal and retroperitoneal) and subcutaneous 

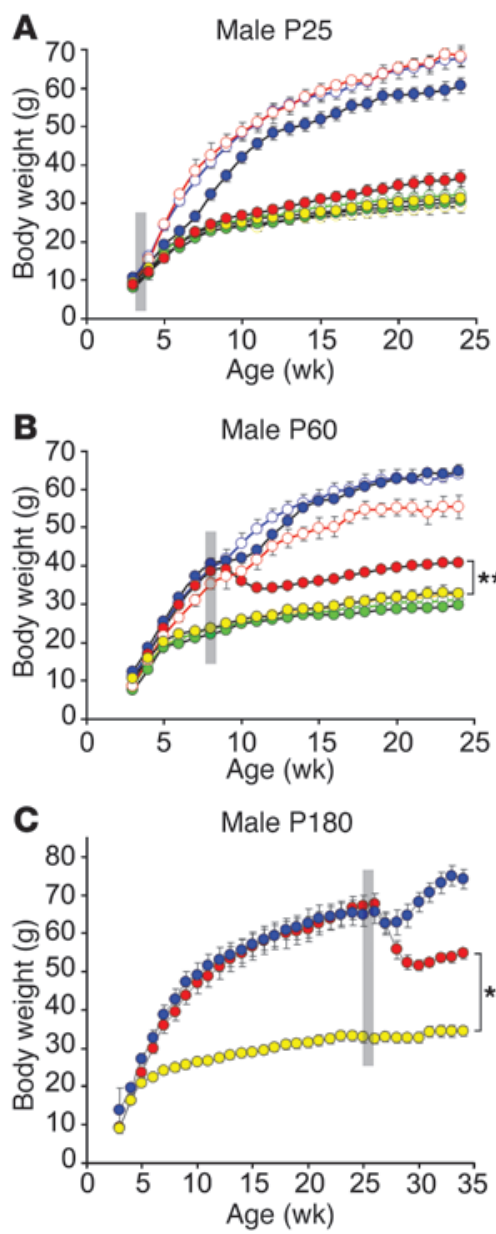
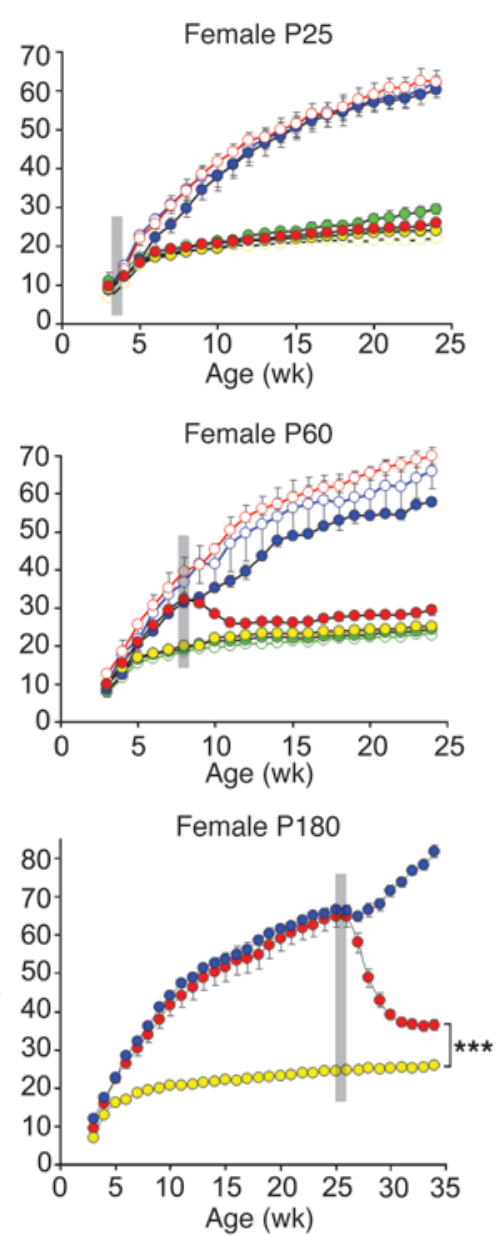

D
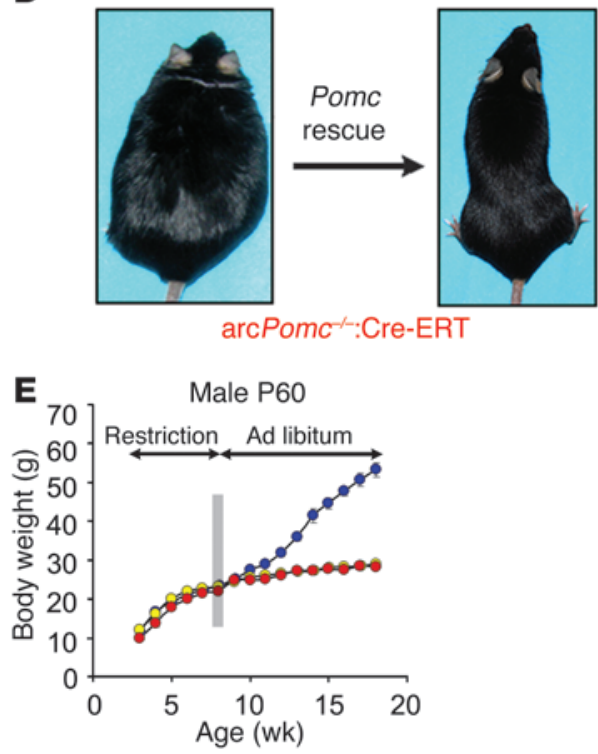

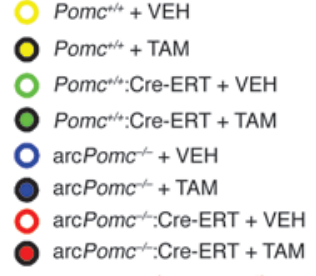

(rescued)

\section{Figure 3}

Response to Pomc rescue in mice with different initial body weights. (A-C) Body weight curves of mice treated at different ages and magnitudes of obesity with either TAM (filled circles) or vehicle (VEH, open circles) as control. Sex and age of treatment are indicated. RMA between Pomc ${ }^{+/+}$and rescued arcPomc ${ }^{-/}:$Cre-ERT, from 12-24 weeks of age (P25 and P60) or from 31-34 weeks of age (P180), significant genotype-treatment (group) effects between Pomc ${ }^{+/+}$and rescued arcPomc ${ }^{-/}$:Cre-ERT at 12-24 weeks of age (P25 and P60) and 31-34 weeks of age (P180) are indicated. Gray bars denote the 5-day TAM or VEH treatment period. Circles represent averages of 3-8 mice. Error bars correspond to SEM. ${ }^{\star \star} P<0.01$, ${ }^{\star \star \star} P<0.001$. (D) A representative P180-treated female mouse before (left) and 8 weeks after (right) treatment with TAM illustrating the marked loss of body mass. (E) Male arcPomc $c^{--}$and arcPomc $c^{---}$:Cre-ERT mice were weight matched to control lean mice by food restriction starting at weaning. The mice were then treated at P60 with TAM and provided chow ad libitum. Yellow circles show the combined average of 5 Pomc $\mathrm{Pol}^{+/+}$and 2 Pomc ${ }^{+/+}:$Cre-ERT mice.

(inguinal) fat stores were normalized in all groups of TAM-treated arcPomc $^{-1}$ :Cre-ERT mice, with the exception of inguinal fat pads in P180-rescued males (Supplemental Figure 3, A and B). Importantly, arcPomc ${ }^{-1-}$ :Cre-ERT rescued mice of all groups showed complete normalization of liver weight due to a remarkable reduction in steatosis (Supplemental Figure 3, A and B). To determine whether weight loss in mice treated at P180 was accompanied by metabolic benefits, despite an incomplete normalization of body weight, we measured serum glucose, insulin, and leptin and calculated the homeostatic model assessment (HOMA) index of insulin resistance before and after Pomc rescue. As shown in Table 1, those parameters greatly improved in arcPomc ${ }^{-1}$ :Cre-ERT mice after body weight loss.

Pomc rescue restored fasting-induced hyperphagia. Finally, we investigated food intake responses to starvation in arcPomc ${ }^{-1-}$ :Cre-ERT mice treated at P180 with TAM. After a 24-hour fast, female $\mathrm{arcPomc}^{-/}$mice failed to exhibit the normal fast-induced hyperphagia triggered in $\mathrm{Pomc}^{+/+}$mice, while male arcPomc ${ }^{-/}$showed a sig- nificantly reduced response (Figure 6 and Supplemental Figure 4). Conversely, TAM-treated arcPomc ${ }^{-/-}$:Cre-ERT mice of both sexes recovered the fast-induced hyperphagic response. The absolute food intake of $\mathrm{Pomc}^{+/+}$and rescued arcPomc ${ }^{-/-}$:Cre-ERT mice during the first 24 hours of refeeding matched the pre-fasting food intake of obese arcPomc ${ }^{-1-}$ mice (Supplemental Figure 4). Overall, these results suggest that the lack of central POMC peptides caused mice to eat as if they were under continuous starvation and, therefore, insensitive to actual food deprivation. Since TAM-treated arcPomc ${ }^{-1}:$ Cre-ERT mice recovered hyperphagia induced by fasting, we conclude that the dynamic role of arcuate POMC neurons as integrators of energy balance circuits is restored in these mice.

\section{Discussion}

By inserting a loxP-flanked neo cassette into the neuronal enhancer module of Pomc, we produced a mouse strain carrying a reversible genetic deficit that allowed us to directly measure the elastic limit 

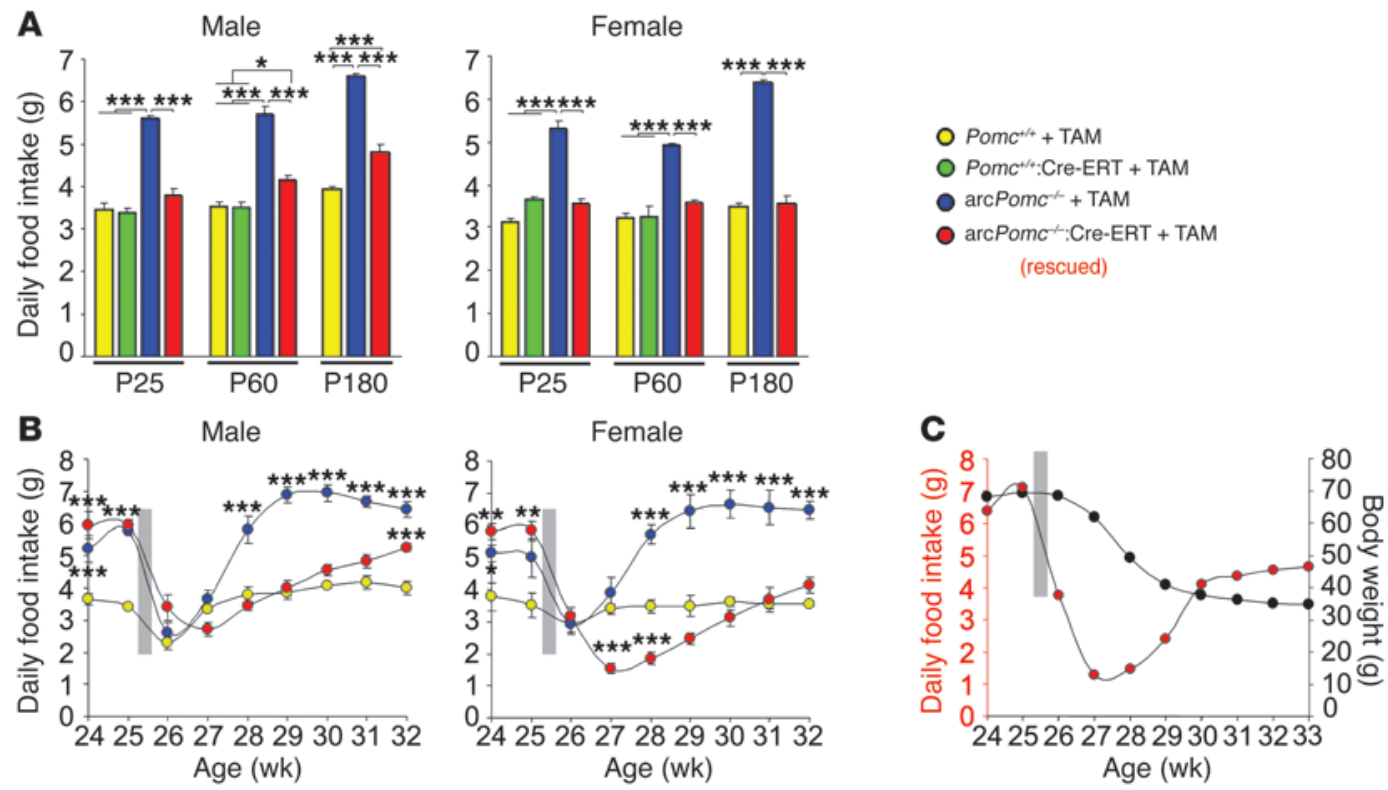

Figure 4

The stage of Pomc rescue determines the magnitude of food intake improvement. (A) Daily food intake of TAM-treated mice after body weight stabilization. (B) Changes in daily food intake before and after treatment of mice with TAM at P180. Only significant results for arcPomc ${ }^{-/-}$and rescued arcPomc ${ }^{-/-}$:Cre-ERT compared with $\mathrm{Pomc}^{+/+}$are indicated. (C) Food intake superimposed with body weight changes in a representative rescued female arcPomc ${ }^{-1}:$ Cre-ERT mouse treated with TAM at P180. Gray bars denote the 5-day TAM treatment period. Circles and colored bars are averages of 3-8 mice. Error bars correspond to SEM. ${ }^{\star} P<0.05,{ }^{\star \star} P<0.01$ and ${ }^{\star * \star} P<0.001$.

of body weight regulation. A major advantage of this novel mouse model is that early-onset severe obesity is caused by inhibiting the expression of a single gene (Pomc), in a specific population of arcuate neurons, which can be reactivated by TAM-induced removal of the interfering neo cassette. An additional advantage of this model of extreme hyperphagia and obesity is that mice only have access to regular chow with low hedonic value, which precludes the sensitization of reward circuits normally activated by highly palatable food used in diet-induced obesity paradigms.

Pomc expression rescue in Arc neurons of weanling mice resulted in complete normalization of subsequent body weight. We ascribe the phenotypic rescue specifically to Arc neurons because of the small number of NTS POMC neurons, the very low basal levels of Pomc mRNA in the NTS relative to Arc, and the demonstration that obese $\operatorname{arcPomc}^{-1-}$ mice retained a substantial fraction of NTS Pomc expression prior to TAM treatment. Our results have several important implications regarding the central POMC system. First, despite the precocious differentiation of POMC neurons in the embryonic hypothalamus (27), POMC peptides are not required during prenatal brain development or in the first 3 weeks of postnatal life to establish a normal body weight set point. Moreover, our results demonstrate that POMC neurons are normally specified during development and properly incorporated into functional neural circuits in the complete absence of POMC peptides, as indicated by the expression of a POMC-EGFP transgene mimicking the expression pattern of Pomc in hypothalamic neurons and the functional rescue of Pomc expression in the adult Arc. The physiological role of hypothalamic POMC prior to P25 remains to be fully defined.

The improvement in body weight of $\operatorname{arcPomc}^{-1-}$ mice was progressively attenuated as the rescue of Pomc expression was delayed after weaning, despite the fact that TAM/CreER-mediated somatic recombination of the floxed-neo Pomc alleles was similar for both sexes and for all ages and body weights at the time of treatment. This finding supports the conclusions drawn from both clinical $(6,7)$ and rodent models $(11-13)$, which relied on high-fat dietinduced obesity, that a condition of chronic overweight leads to secondary adaptations in the central regulation of energy homeostasis that act to perpetuate obesity despite reductions in caloric intake. Furthermore, since we controlled for treatment, genetic, and environmental factors by rescuing central Pomc expression in inbred mouse siblings fed a standard chow of low hedonic value, our results show for the first time to our knowledge that obesity is a primary condition that permanently alters the normal body weight set point by imposing a maladaptive allostatic state that ultimately defends a greater body weight. This conclusion is supported by our demonstration that prevention of excessive weight gain by calorie restriction in arcPomc ${ }^{-1-}$ :CreERT mice extended the time window for complete normalization of subsequent body weight by Pomc rescue. Although the final body weight reached by extremely obese mice after treatment was still $40 \%-60 \%$ greater than that of normal lean mice, the reduction in weight was sufficient to significantly reduce comorbidities associated with obesity. This phenomenon is usually seen in severely obese patients who lose approximately $10 \%$ or more of their weight (28). However, it should be noted that the improvement in glucose homeostasis could also be due to weight loss-independent actions of the restored melanocortin peptides in the brain. The activation of MC4 receptors selectively reexpressed on cholinergic sympathetic preganglionic neurons has been demonstrated to be sufficient to attenuate hyperglycemia and hyperinsulinemia without affecting food intake or adiposity (29). 
A

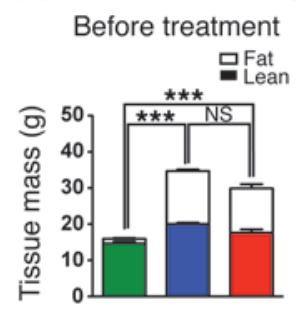

B

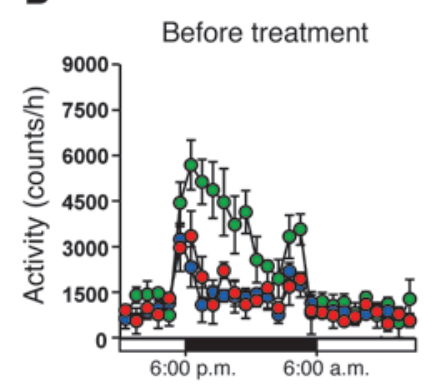

C

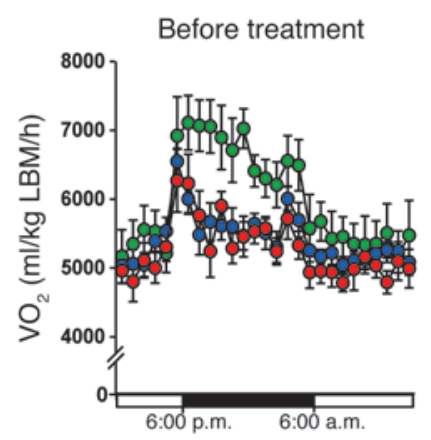

Female P60

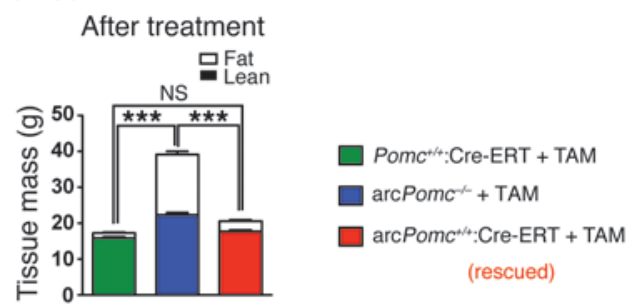

Female P60

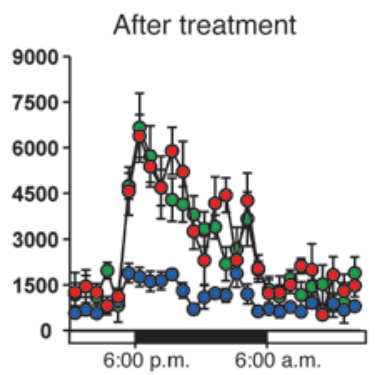

Female P60

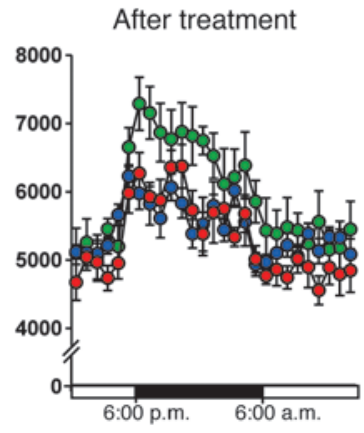

12-h dark period - Before treatment NS

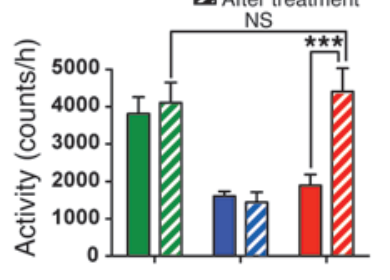

12-h dark period Before treatment $\star \star$

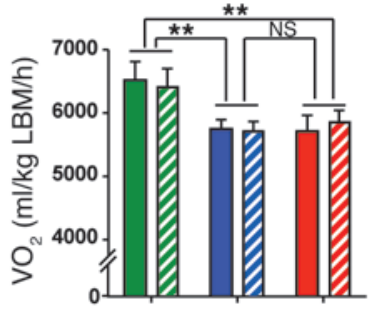

\section{Figure 5}

Serial measurements of metabolic parameters before and 4 weeks after TAM treatment in a cohort of female P60 mice. (A) Body composition measured by NMR. ${ }^{* * *} P<0.001$, pairwise comparison of body fat by Bonferroni's post hoc test. (B) Total locomotor activity in the horizontal plane measured by infrared beam breaks in the CLAMS chambers. The line graphs show average hourly activity counts over the final 24-hour period of a 72-hour continuous measurement. The dark period between 6 p.m. and 6 a.m. is indicated by the black bar below the $x$ axis. The bar graph on the right shows the average hourly activity over the entire 12-hour dark period. ${ }^{* \star}{ }^{\star \star} P<0.001$, effect of TAM treatment in arcPomc $c^{-1-}$ :Cre-ERT mice. (C) $\mathrm{VO}_{2}$ corrected to lean body mass (LBM) determined by indirect calorimetry in the CLAMS chambers. The line graphs show average hourly $\mathrm{VO}_{2}$ over the final 24-hour period of a 72-hour continuous measurement. The dark period between 6 p.m. and 6 a.m. is indicated by the black bar under the $x$ axis. The bar graph on the right shows the average hourly $\mathrm{VO}_{2}$ over the entire 12-hour dark period. ${ }^{\star} P<0.01$, pairwise comparison by Bonferroni's post hoc test. None of the 3 genotypes of mice showed a significant change in $\mathrm{VO}_{2}$ after TAM treatment. All data shown are the mean \pm SEM; $n=6-8$ per genotype.
The two notable parameters associated with weight loss after the induction of hypothalamic Pomc were reduction in food consumption and normalization of locomotor activity. The immediate phase of extreme hypophagia lasting for 2-3 weeks after induction of Pomc expression conceivably was due to MCR supersensitivity resulting from the previous lack of melanocortin signaling (30). Energy expenditure, as measured by oxygen consumption, was not altered after Pomc rescue in either sex and actually remained significantly decreased in female mice, despite their increased locomotor activity. It is conceivable that a reduction in diet-induced thermogenesis accompanying the decreased food intake of the rescued mice obscured the expected increase in energy expenditure from the increased locomotion. However, our study was not designed to accurately discriminate between these two components of thermogenesis and basal metabolic rate. Recent reviews have also emphasized the complexity of relying on indirect calorimetry to evaluate thermogenesis in mutant mice that vary significantly in body composition from control animals or over time, as in the present study $(31,32)$. Therefore, the role played by hypothalamic POMC in the regulation of food intake was clearly restored after Pomc rescue, while its role in the control of energy expenditure was apparently resistant to treatment. This could be a consequence of different pathways mediating both roles of melanocortin peptides through MC4 receptors $(29,33)$. Alternatively, the reduction in oxygen consumption could be an indirect consequence of obesity rather than a direct effect of Pomc deficiency (34). In either case, the drop in energy expenditure contributes to the inability to restore a normal body weight set point.

The loss and restoration of $\beta$-endorphin, in parallel with those of the melanocortin peptides, likely also play a role in the body weight phenotype of these mice. We previously showed that mice with a selective absence of $\beta$-endorphin develop a mild obesity phenotype, particularly in males (35). Furthermore, a recent study suggested that an endogenous opioid, likely $\beta$-endorphin released from POMC neurons, inhibits hypothalamic AgRP neurons (36). Therefore, the absence of $\beta$-endorphin in arcPomc ${ }^{-/-}$mice may lead to a disinhibition of AgRP neurons that would complement the absence of melanocortin peptides and combine to induce severe hyperphagia.

Obese $\operatorname{arcPomc}^{-1-}$ mice were hyperleptinemic before treatment, a condition probably leading to partial leptin resistance (37). There was a direct correlation between serum leptin levels before treatment (not shown for mice treated at P60), the weight at the time of Pomc rescue, the extent of hypophagia after treatment, and the percentage of weight loss. Furthermore, the restoration of fast-induced overfeeding suggests the reestablishment of Pomc responses to leptin in rescued arcPomc $^{-1-}$ :Cre-ERT mice, since this adaptation has been shown to be mediated, at least in part, by leptin activation of Stat3 (38). On the other hand, we found an inverse correlation between weight at the time of treatment and the subsequent levels of hypothalamic Pomc mRNA. Mice rescued at P25, which were lean at the moment 
Table 1

Hyperglycemia, hyperinsulinemia, HOMA index, and hyperleptinemia are improved after Pomc restoration

\begin{tabular}{|c|c|c|c|c|c|c|c|c|c|}
\hline \multirow[t]{2}{*}{ Sex } & \multirow[t]{2}{*}{ Genotype } & \multicolumn{2}{|c|}{ Glucose (mg/dl) } & \multicolumn{2}{|c|}{ Insulin (ng/ml) } & \multicolumn{2}{|c|}{ HOMA index } & \multicolumn{2}{|c|}{ Leptin (ng/ml) } \\
\hline & & Before & After & Before & After & Before & After & Before & After \\
\hline Male & 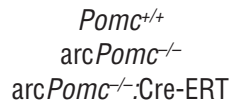 & $\begin{array}{l}108 \pm 4 \\
128 \pm 7 \\
162 \pm 8\end{array}$ & $\begin{array}{l}121 \pm 6 \\
137 \pm 2 \\
126 \pm 7 \mathrm{E}\end{array}$ & $\begin{array}{l}0.31 \pm 0.09 \\
2.33 \pm 0.27 \\
2.94 \pm 0.62^{A}\end{array}$ & $\begin{array}{l}0.43 \pm 0.13 \\
2.96 \pm 0.58^{A} \\
1.81 \pm 0.37\end{array}$ & $\begin{aligned} 2.0 & \pm 0.5 \\
18.4 & \pm 2.8 \\
28.4 & \pm 2.3 \mathrm{D}\end{aligned}$ & $\begin{array}{c}3.2 \pm 1 \\
24.5 \pm 4.4^{A} \\
13.5 \pm 2.3\end{array}$ & $\begin{array}{c}5.1 \pm 1.3 \\
137.4 \pm 6.6^{\mathrm{B}} \\
156.6 \pm 11.0^{\mathrm{B}}\end{array}$ & $\begin{aligned} 4.9 & \pm 1.6 \\
178.2 & \pm 22.2^{\mathrm{B}, \mathrm{C}} \\
53.0 & \pm 12.8^{\mathrm{A}, \mathrm{E}}\end{aligned}$ \\
\hline Female & 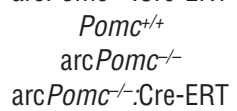 & $\begin{array}{l}110 \pm 2 \\
161 \pm 8^{D} \\
161 \pm 12^{D}\end{array}$ & $\begin{aligned} 111 & \pm 2 \\
130 & \pm 3^{A} \\
80 & \pm 2^{\mathrm{E}}\end{aligned}$ & $\begin{array}{l}0.18 \pm 0.02 \\
2.39 \pm 0.07^{D} \\
2.29 \pm 0.26^{B}\end{array}$ & $\begin{array}{l}0.26 \pm 0.06 \\
2.08 \pm 0.47^{\mathrm{A}} \\
1.69 \pm 0.5^{\mathrm{A}}\end{array}$ & $\begin{array}{c}1.2 \pm 0.1 \\
23.5 \pm 1.2^{\mathrm{B}} \\
23.0 \pm 2.6^{\mathrm{B}}\end{array}$ & $\begin{aligned} 1.7 & \pm 0.4 \\
16.4 & \pm 2.7^{\mathrm{A}} \\
8.4 & \pm 2.6^{\mathrm{C}}\end{aligned}$ & $\begin{array}{c}5.2 \pm 1.4 \\
221.9 \pm 22.7^{B} \\
180.1 \pm 18.9^{B}\end{array}$ & $\begin{aligned} 6.4 & \pm 0.9 \\
199.6 & \pm 26.1^{\mathrm{B}} \\
28.1 & \pm 4.2^{\mathrm{E}}\end{aligned}$ \\
\hline
\end{tabular}

Serum levels of glucose, insulin, and leptin in fasted mice were determined 1 week before and 10-11 weeks after treatment with TAM at P180. The HOMA index of insulin resistance was calculated using the formula (blood glucose [mmol/l] $) \times($ serum insulin $[\mathrm{mU} / / \mathrm{l}]) / 22.5$. ${ }^{\mathrm{A}} P<0.05,{ }^{\mathrm{B}} P<0.001,{ }^{\mathrm{D}} P<0.01 \mathrm{versus}$ $\mathrm{Pomc}^{+/+}$mice of the same age and sex (1-way ANOVA). ${ }^{C P}<0.05$, EP $<0.001$, pairwise comparison, after versus before treatment within the same group (RMA, Bonferroni's post hoc test). Values represent mean $\pm \operatorname{SEM}(n=4-6)$.

of treatment and presumably normoleptinemic, recovered more than $50 \%$ of Pomc mRNA levels and defended a normal body weight. In contrast, mice rescued at P60 or P180 after the development of obesity and hyperleptinemia had attenuated Pomc expression below the threshold capable of sustaining normal satiety $(<50 \%)$, consistent with a secondary phenotypic influence of leptin resistance, and therefore defended a higher body weight set point. This and other mechanisms such as the failure to normalize energy expenditure would contribute to the progressive waning of body weight normalization associated with treatment delay.

A recurring aspect of our study was the sexual dimorphism in treatment responses. Female mice had greater improvement in food intake and body weight. Both humans and rodents show sex-associated dif- ferences in fat distribution and leptin secretion (39). As females are more sensitive to leptin than males (40), it is possible that the hypophagia and body weight loss elicited by POMC immediately after rescue are influenced by leptin action (through POMC cells), although with greater response in females. It is likely that estrogen's anorectic effect is also responsible for this dimorphism, since it is known that i.c.v. estradiol injection induces anorexia and increases excitatory synapses on POMC cells (41). In addition, we previously found that estrogen receptor $\alpha$ (ESR1) is a candidate nuclear receptor factor to regulate neuronal POMC expression, since it is expressed in POMC neurons during development and adulthood (42). Finally, although not evaluated in the present study, it is also possible that alterations in the posttranslational processing of POMC to its bioactive peptides

\section{Figure 6}

Pomc rescue restores compensatory hyperphagia induced by food deprivation. Male and female mice treated with TAM at P180 were subjected to 24 hours of food deprivation at 33-35 weeks of age (7-9 weeks after TAM treatment). Food was then returned to the cages (refeeding period), and ad libitum intake was measured at 2, 8, 24, 48, and 72 hours after refeeding. Each bar represents average of food taken by mice of a genotype group $(n=5-7)$ during the indicated period, expressed as a percentage of their average individual pre-fasting daily food consumption. The corresponding raw data are shown in Supplemental Figure 4. Pre-fasting data represent average daily food intake of the same mice during 4 consecutive days before fasting. Error bars correspond to SEM. ${ }^{\star} P<0.05,{ }^{* \star} P<0.01$, ${ }^{* * *} P<0.001$ (OWA).
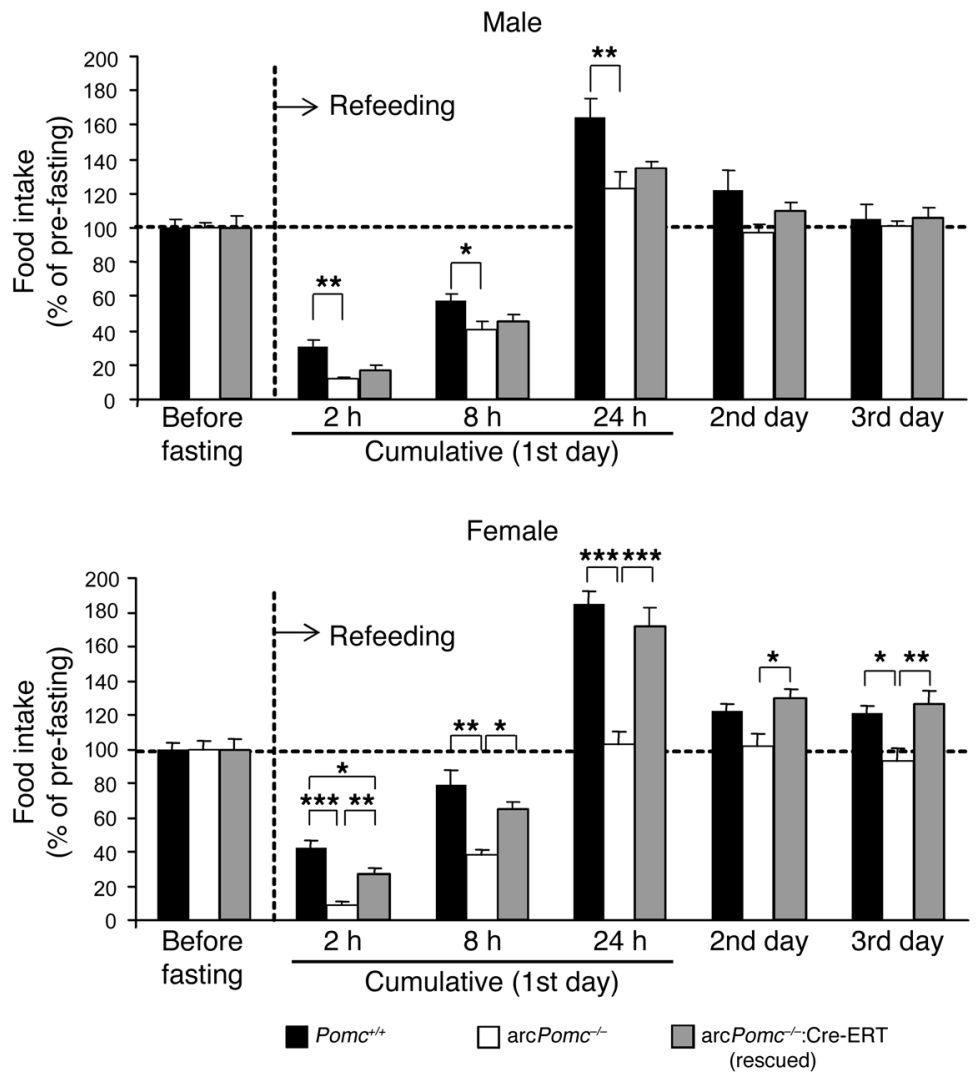
in association with sex, aging, and preceding obesity status contribute to the allostatic state following the restoration of Pomc expression.

In conclusion, our findings show that obesity is a self-perpetuating condition and reinforce the importance of early consultation and weight management in children to prevent obesity, especially when taking into account that the probability of adult obesity exceeds $50 \%$ in people who were overweight at 6 years of age (43).

\section{Methods}

\section{Animal care}

Mice were housed in ventilated cages under controlled temperature and photoperiod (12-hour light/ 12-hour dark cycle, lights on from 6 a.m. to 6 p.m.), with tap water and laboratory chow containing $28.0 \mathrm{kcal} \%$ protein, $12.1 \mathrm{kcal} \%$ fat, and $59.8 \mathrm{kcal} \%$ carbohydrate available ad libitum.

\section{Generation and breeding of mice}

ArcPomc. Mice carrying a floxed neo-cassette inserted in the neuronal Pomc enhancer neighborhood were generated as follows: The targeting vector was constructed with genomic sequences between -13 and $-6.5 \mathrm{~kb}$ of mouse Pomc that were previously isolated (19) and a transgene construct harboring a 172-bp deletion of Pomc neuronal enhancer nPE2 (18). A neomycinresistance cassette (PGK-neo) flanked by loxP sites was inserted $40 \mathrm{bp}$ upstream of the deleted nPE2 region (10.3 kb upstream of the Pomc transcriptional start site). See Supplemental Methods for more details.

$\mathrm{ArcPomc}^{-1-}: \mathrm{Cre}-\mathrm{ERT}$. ArcPomc ${ }^{+/-}$mice were crossed with Cre-ERT mice (25) (B6.Cg-Tg[cre/Esr1] $]^{5 \mathrm{~mm} / J}$; The Jackson Laboratory), previously backcrossed with C57BL/6J for 5 generations, to obtain $\operatorname{arcPomc}^{+/}$:Cre-ERT mice. Thereafter, $\operatorname{arcPomc} c^{+-}:$Cre-ERT were mated with $\operatorname{arcPomc}{ }^{+/-}$mice to obtain all control and treated groups.

\section{General study design}

Three cohorts of mice were generated by breeding strategies described above and treated at different ages: postnatal days 25,60 , or $180 \pm 3$ days (P25, P60, and P180, respectively). A fourth cohort of male arcPomc ${ }^{-1-}$ and $\operatorname{arcPomc}^{-1}$ :Cre-ERT mice was subjected to food restriction from the time of weaning to match body weights of $\mathrm{Pomc}^{+/+}$and $\mathrm{Pomc}^{+/+}$:Cre-ERT lean control mice. After TAM treatment at P60, they were allowed to consume food ad libitum. All mice were individually housed within the first week after weaning and weighed twice a week at 3 p.m. At the indicated age, mice were treated either with 5 consecutive daily i.p. injections of $50 \mathrm{mg} / \mathrm{kg} / \mathrm{d}$ TAM (Sigma-Aldrich) prepared as described previously (44) or with the oil vehicle as control. Food intake was calculated by weighing food from cages every day from Monday through Friday ( 3 p.m.), from 2 weeks before treatment (P60 and P180) until the fourth week after treatment (P25, P60, P180), and during an additional 2 weeks before ending experiments. Blood samples were collected 1 week before (P60, P180) treatment and 1 week before ending experiments (P25, P60, P180). Six weeks after treatment, P180-treated mice were subjected to a 24-hour fast ( 9 a.m. -9 a.m.) and then were allowed to eat ad libitum, and food was weighed after 0, 2, 8, 24, 48, and 72 hours during the refeeding period. Daily food intake during the previous week was used as a reference. Most of the mice were killed by cervical dislocation or decapitation at 5-6 months of age (P25 and P60) or 8-9 months of age (P180), individual fat pads and other organs were weighed, and the hypothalamus was snap frozen for later RNA extraction. The remaining mice were anesthetized and perfused with $4 \%$ paraformaldehyde for subsequent immunohistochemistry.

A fifth cohort of P60 arcPomc ${ }^{-1 /}$ :Cre-ERT:POMC-EGFP mice was generated for a repeated measures study of energy expenditure by indirect calorimetry $\left(\mathrm{VO}_{2}, \mathrm{VCO}_{2}\right)$ and locomotor activity using a Comprehensive Lab Animal Monitoring System (CLAMS) (Columbus Instruments). These mice were individually housed starting at 8 weeks of age and treated with TAM as above at P70 \pm 6 days. Data were collected from the mice in the CLAMS units for 3 days immediately before TAM treatment and for a second 3-day period, 5 weeks later. Oxygen consumption $\left(\mathrm{VO}_{2}\right)$ was corrected for lean body mass determined by body composition analysis using NMR (Minispec LF9011, Bruker) immediately after each CLAMS session.

\section{Immunobistochemistry}

Vibratome sections $(50 \mu \mathrm{m})$ of paraformaldehyde-perfused brains were subjected to immunohistochemistry with an anti-rat ACTH antibody $(1: 10,000$, A.F. Parlow, National Hormone and Peptide Program, Harbor-UCLA Medical Center, Torrance, California, USA) and developed with diaminobenzidine (DAB; Sigma-Aldrich) or detected by Alexa Fluor 555-coupled anti-rabbit IgG (Molecular Probes, Invitrogen). Images were taken with an Olympus DP72 digital camera connected to an upright research microscope (Olympus BX51). The number of DAB-stained POMC neurons (positive for ACTH) in rescued mice was estimated all across the Arc and matched with equivalent slices of TAM-treated $\mathrm{Pomc}^{+/+}$mice to calculate percentage of recovery. For immunofluorescence, ACTH-positive cells (red) were colocalized with endogenous EGFP fluorescence (green). E14 sections were developed with SG staining (Vector). See Supplemental Methods for more details.

\section{Statistics}

All data presented are the mean \pm SEM and were analyzed by RMA or OWA unless otherwise stated using Statistica (version 7). Post hoc pairwise comparisons between groups were performed by Bonferroni's post hoc test. $P$ values less than 0.05 were considered significant.

\section{Study approval}

All procedures were approved by the Institutional Animal Care and Use Committees and followed the Public Health Service guidelines for the humane care and use of experimental animals.

\section{Acknowledgments}

We thank R. Kruse Bend, B. Warren, and V. Rodríguez for valuable technical support and N. Qi for analysis of calorimetry data. This work was supported by NIH grants DK066604 (to M.J. Low) and DK068400 (to M.J. Low and M. Rubinstein); International Research Scholar Grant of the Howard Hughes Medical Institute (HHMI) (to M. Rubinstein); Agencia Nacional de Promoción Científica y Tecnológica, Argentina (to V.F. Bumaschny and M. Rubinstein); Consejo Nacional de Investigaciones Científicas y Técnicas, Argentina (to V.F. Bumaschny, F.S.J. de Souza, and M. Rubinstein); and Universidad de Buenos Aires. This work utilized core services provided by the Oregon Health and Science University Transgenic Core Laboratory and the University of Michigan Animal Phenotyping Core supported by the Michigan Diabetes Research and Training Center and the Michigan Nutrition and Obesity Research Center (NIH grants DK020572 and DK089503).

Received for publication December 23, 2011, and accepted in revised form August 20, 2012.

Address correspondence to: Malcolm J. Low, Department of Molecular and Integrative Physiology, 6116 Brehm, 1000 Wall St., University of Michigan, Ann Arbor, Michigan 48105, USA. Phone: 734.647.1350; Fax: 734.232.8175; E-mail: mjlow@umich.edu. Or to: Marcelo Rubinstein, INGEBI-CONICET, Vuelta de Obligado 2490, C1428ADN Buenos Aires, Argentina. Phone: 5411.47832871; Fax: 5411.47868578; E-mail: mrubins@dna.uba.ar. 
1. WHO. Factsheetno. 311: Obesity and Overweight. Geneva, Switzerland: World Health Organization; 2011.

2. Haslam DW, James WP. Obesity. Lancet. 2005; 366(9492):1197-1209.

3. Leibel RL. Molecular physiology of weight regulation in mice and humans. Int J Obes (Lond). 2008; 32(suppl 7):S98-S108.

4. Bouchard C, et al. Overfeeding in identical twins: 5-year postoverfeeding results. Metabolism. 1996; 45(8):1042-1050

5. Pasquet P, Apfelbaum M. Recovery of initial body weight and composition after long-term massive overfeeding in men. Am J Clin Nutr. 1994; 60(6):861-863.

6. Rosenbaum M, et al. Low-dose leptin reverses skeletal muscle, autonomic, and neuroendocrine adaptations to maintenance of reduced weight J Clin Invest. 2005;115(12):3579-3586.

7. Tremblay A, Chaput JP. Adaptive reduction in thermogenesis and resistance to lose fat in obese men. BrJ Nutr. 2009;102(4):488-492.

8. Bray GA, Greenway FL. Pharmacological treatment of the overweight patient. Pharmacol Rev. 2007; 59(2):151-184.

9. Kraschnewski JL, et al. Long-term weight loss maintenance in the United States. Int J Obes (Lond). 2010 34(11):1644-1654.

10. Sturm R. Increases in morbid obesity in the USA: 2000-2005. Public Health. 2007;121(7):492-496.

11. Rolls BJ, Rowe EA, Turner RC. Persistent obesity in rats following a period of consumption of a mixed, high energy diet. J Physiol. 1980;298:415-427.

12. Levin BE, Dunn-Meynell AA. Defense of body weight against chronic caloric restriction in obesity-prone and -resistant rats. Am J Physiol Regul Integr Comp Physiol. 2000;278(1):R231-R237.

13. Guo J, Jou W, Gavrilova O, Hall KD. Persistent dietinduced obesity in male $\mathrm{C} 57 \mathrm{BL} / 6$ mice resulting from temporary obesigenic diets. PLoS One. 2009; 4(4):e5370.

14. Schulkin J. Allostasis: a neural behavioral perspective. Horm Behav. 2003;43(1):21-27.

15 . Woods SC. The control of food intake: behavioral versus molecular perspectives. Cell Metab. 2009, 9(6):489-498.

16. Coll AP, Farooqi IS, O'Rahilly S. The hormonal control of food intake. Cell. 2007;129(2):251-262.

17. de Souza FS, Bumaschny VF, Low MJ, Rubinstein M. Subfunctionalization of expression and peptide domains following the ancient duplication of the proopiomelanocortin gene in teleost fishes. $\mathrm{Mol}$ Biol Evol. 2005;22(12):2417-2427.
18. de Souza FS, et al. Identification of neuronal enhancers of the proopiomelanocortin gene by transgenic mouse analysis and phylogenetic footprinting. Mol Cell Biol. 2005;25(8):3076-3086.

19. Cowley MA, et al. Leptin activates anorexigenic POMC neurons through a neural network in the arcuate nucleus. Nature. 2001;411(6836):480-484.

20. Benoit SC, et al. The catabolic action of insulin in the brain is mediated by melanocortins. J Neurosci. 2002; 22(20):9048-9052.

21. Yaswen L, Diehl N, Brennan MB, Hochgeschwender U. Obesity in the mouse model of proopiomelanocortin deficiency responds to peripheral melanocortin. Nat Med. 1999;5(9):1066-1070.

22. Krude H, Biebermann H, Luck W, Horn R, Brabant G, Gruters A. Severe early-onset obesity, adrenal insufficiency and red hair pigmentation caused by POMC mutations in humans. Nat Genet. 1998 19(2):155-157

23. Johnson PM, Kenny PJ. Dopamine D2 receptors in addiction-like reward dysfunction and compulsive eating in obese rats. Nat Neurosci. 2010 13(5):635-641.

24. Lakso M, et al. Efficient in vivo manipulation of mouse genomic sequences at the zygote stage. Proc Natl Acad Sci U S A. 1996;93(12):5860-5865.

25. Hayashi S, McMahon AP. Efficient recombination in diverse tissues by a tamoxifen-inducible form of Cre: a tool for temporally regulated gene activation/inactivation in the mouse. Dev Biol. 2002 244(2):305-318.

26. Bjorbaek C. Central leptin receptor action and resistance in obesity. J Investig Med. 2009;57(7):789-794.

27. Santangelo AM, de Souza FS, Franchini LF, Bumaschny VF, Low MJ, Rubinstein M. Ancient exaptation of a CORE-SINE retroposon into a highly conserved mammalian neuronal enhancer of the proopiomelanocortin gene. PLoS Genet. 2007; 3(10):1813-1826.

28. Knowler WC, et al. Reduction in the incidence of type 2 diabetes with lifestyle intervention or metformin. N Engl J Med. 2002;346(6):393-403.

29. Rossi J, et al. Melanocortin-4 receptors expressed by cholinergic neurons regulate energy balance and glucose homeostasis. Cell Metab. 2011;13(2):195-204.

30. Tolle V, Low MJ. In vivo evidence for inverse agonism of Agouti-related peptide in the central nervous system of proopiomelanocortin-deficient mice. Diabetes. 2008;57(1):86-94.

31. Butler AA, Kozak LP. A recurring problem with the analysis of energy expenditure in genetic models expressing lean and obese phenotypes. Diabetes.
2010;59(2):323-329.

32. Kaiyala KJ, Schwartz MW. Toward a more complete (and less controversial) understanding of energy expenditure and its role in obesity pathogenesis. Diabetes. 2011;60(1):17-23.

33. Balthasar N, et al. Divergence of melanocortin pathways in the control of food intake and energy expenditure. Cell. 2005;123(3):493-505.

34. Weide $\mathrm{K}$, et al. Hyperphagia, not hypometabolism, causes early onset obesity in melanocortin-4 receptor knockout mice. Physiol Genomics. 2003; 13(1):47-56.

35. Appleyard SM, et al. A role for the endogenous opioid beta-endorphin in energy homeostasis. Endocrinology. 2003;144(5):1753-1760.

36. Yang Y, Atasoy D, Su HH, Sternson SM. Hunger states switch a flip-flop memory circuit via a synaptic AMPK-dependent positive feedback loop. Cell. 2011;146(6):992-1003.

37. Myers MG, Cowley MA, Munzberg H. Mechanisms of leptin action and leptin resistance. Annu Rev Physiol. 2008;70:537-556.

38. Xu AW, Ste-Marie L, Kaelin CB, Barsh GS. Inactivation of signal transducer and activator of transcription 3 in proopiomelanocortin (Pomc) neurons causes decreased pomc expression, mild obesity, and defects in compensatory refeeding. Endocrinology. 2007;148(1):72-80.

39. Woods SC, Gotoh K, Clegg DJ. Gender differences in the control of energy homeostasis. Exp Biol Med (Maywood). 2003;228(10):1175-1180.

40. Clegg DJ, Riedy CA, Smith KA, Benoit SC, Woods SC. Differential sensitivity to central leptin and insulin in male and female rats. Diabetes. 2003;52(3):682-687.

41. Gao Q, et al. Anorectic estrogen mimics leptin's effect on the rewiring of melanocortin cells and Stat3 signaling in obese animals. Nat Med. 2007; 13(1):89-94.

42. de Souza FS, Nasif S, Lopez-Leal R, Levi DH, Low MJ, Rubinsten $M$. The estrogen receptor alpha colocalizes with proopiomelanocortin in hypothalamic neurons and binds to a conserved motif present in the neuron-specific enhancer nPE2. Eur JPharmacol. 2011;660(1):181-187.

43. Whitaker RC, Wright JA, Pepe MS, Seidel KD, Dietz WH. Predicting obesity in young adulthood from childhood and parental obesity. NEngl J Med. 1997; 337(13):869-873

44. Metzger D, Chambon P. Site- and time-specific gene targeting in the mouse. Methods. 2001; 24(1):71-80. 